\title{
Set-based analysis of the variable-geometry suspension system
}

\author{
Balázs Németh* Péter Gáspár** \\ * Institute for Computer Science and Control, Hungarian Academy of \\ Sciences, Hungary; Kende u. 13-17, H-1111 Budapest, Hungary; \\ Phone: +36-12796173; E-mail: bnemeth@sztaki.hu \\ ** Institute for Computer Science and Control, Hungarian Academy of \\ Sciences, MTA-BME Control Engineering Research Group, Hungary; \\ Fax:+36-14667503; Phone:+36-12796171; E-mail: gaspar@sztaki.hu
}

\begin{abstract}
The paper gives an analysis of a variable-geometry suspension system. Since the system also affects both the wheel camber angle and the additional steering angle, the coordination of steering and wheel tilting can be handled by this system. Since the nonlinear effects of the system are significant, the paper gives a detailed set-based analysis for the possibilities and constrains. A nonlinear polynomial SOS (Sum-of-Squares) programming method is applied to present the advantages of the variable-geometry suspension system.
\end{abstract}

Keywords: Variable-geometry suspension; Performance analysis; SOS method; Reconfigurable control.

\section{INTRODUCTION AND MOTIVATION}

The variable-geometry suspension system is a mechanism with which road holding can be improved, see Evers et al. [2008]. Several papers for various kinematic models of suspension systems have been published. A nonlinear model of the McPherson strut suspension system was published by Fallah et al. [2009], Németh and Gáspár [2012]. By using this model the kinematic parameters, such as camber, caster and king-pin angles, were examined. The kinematic design of a double-wishbone suspension system was examined by Sancibrian et al. [2010]. The vehicle-handling characteristics based on a variable roll center suspension were proposed by Lee et al. [2008]. A rear-suspension active toe control for the enhancement of driving stability was proposed by Goodarzia et al. [2010].

Another field of variable-geometry suspension is the steering of narrow vehicles, see Piyabongkarn et al. [2004]. These vehicles require the design of an innovative active wheel tilt and steer control strategies in order to perform steering similarly to a car on straight roads but in bends they tilt as motorcycles, see Suarez [2012]. The active tilt control system, which assists the driver in balancing the vehicle and performs tilting in the bend, is an essential part of a narrow vehicle system, see Piyabongkarn et al. [2004].

In the paper the coordination of steering and wheel tilting is analyzed. The efficiency of variable-geometry suspension has been presented in preliminary works. Németh and Gáspár [2013b] proposes the interaction between the

\footnotetext{
* The research has been conducted as part of the project TÁMOP4.2.2.A-11/1/KONV-2012-0012: Basic research for the development of hybrid and electric vehicles. The Project is supported by the Hungarian Government and co-financed by the European Social Fund.
}

steering and the camber angle, which is ensured by the suspension construction. A control and construction design method guarantees the optimal utilization of the maximum control forces on the tires. Although the reachable set analysis of a linear vehicle model can be a relatively fast and easily applicable technique for actuator intervention limit determination, it has some drawbacks as it is shown in the following example.

\section{Motivation example}

In Figure 1 the reachable sets of a variable-geometry suspension are illustrated using a linear method. Two scenarios are compared in the analysis: the reachable front and rear side-slips $\alpha_{1}, \alpha_{2}$ with limited steering and tilting actuation are computed at two vehicles. In first vehicle pure steering actuation is considered, while in the second vehicle steering and wheel tilting are simultaneously actuated by variable-geometry suspension. The illustration shows that the difference between the two scenarios is quite small. Thus, the effect of wheel tilting can be neglected. In spite of the reachable set analysis, Németh and Gáspár [2013a] proposes that the modification of the wheel camber angle has a significant advantage in vehicle control. Thus, in this example the efficiency of the variable-geometry suspension in vehicle dynamics is not handled adequately by the linear analysis.

Another drawbacks of the linear analysis is that the nonlinear tire characteristics are ignored, which affects vehicle stability. In a linear analysis the vehicle system is described by the state-space representation $\dot{x}=A x+B_{1} w+B_{2} u$, where matrix $A$ gives information about the stability of the system. Although $A$ depends on longitudinal velocity $v$, the increase of $v$ does not cause instability in the normal operational range of the vehicle. The analysis presented in Figure 1 shows that the reachable sets of the system 


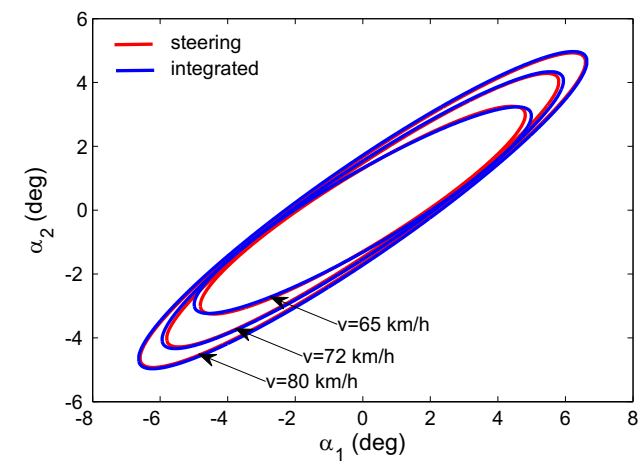

Fig. 1. Reachable sets of the systems

increase at higher $v$. However, the stability margin of the lateral vehicle model is reduced with increasing $v$, see Pacejka [2004]. Consequently, the instability problems at high velocity cannot be analyzed effectively using linear reachable set computation.

In several automotive applications the linearized model of lateral dynamics is sufficient to design controllers for driver assistance systems. However, the comprehensive analysis of vehicle actuator efficiency requires nonlinear techniques to understand the intervention of the systems in detail. In this paper the nonlinear polynomial Sum-of-Squares (SOS) programming method is applied to propose the controloriented advantages of the variable-geometry suspension.

The paper is organized as follows. In Section 2 the nonlinear polynomial vehicle model and the variable-geometry suspension are formulated. The method of SOS-based Maximal Controlled Invariant Set computation is proposed in Section 3. The analysis of the invariant sets at different velocities is a novel design criterion is found in Section 4. Section 5 contains some concluding remarks.

\section{MODELING OF LATERAL VEHICLE DYNAMICS}

In the section the nonlinear lateral vehicle model is presented (see Figure 2), on which the analysis of the actuator efficiency is based. The variable-geometry suspension has different effects on the wheels: modification of steering and camber angles. The relationship between the angles is determined by the construction of the suspension. In this section two system models are detailed. First, the formulation of lateral vehicle dynamics is proposed based on the polynomial approximation of tire model. Second, the modeling and analysis of variable-geometry are presented.

\subsection{Formulation of nonlinear vehicle model}

Modeling tire forces is a crucial point of vehicle dynamics. Several tire models have been published, see examples Pacejka [2004], Kiencke and Nielsen [2000], de Wit et al. [1995], Dugoff et al. [1969]. These models formulate the nonlinearity of longitudinal and lateral tire forces accurately. In the paper a polynomial tire modeling approach is presented.

The lateral dynamics of the vehicle is formulated by the following dynamic model:

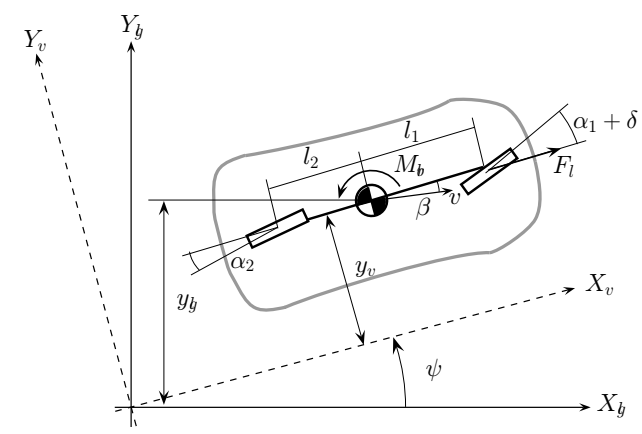

Fig. 2. Scheme of lateral vehicle model

$$
\begin{aligned}
J \ddot{\psi} & =\mathbf{F}_{\text {lat }, \mathbf{1}}\left(\alpha_{1}\right) l_{1}-\mathbf{F}_{\text {lat }, \mathbf{2}}\left(\alpha_{2}\right) l_{2}= \\
& =\mathcal{F}_{1}\left(\alpha_{1}\right) l_{1}-\mathcal{F}_{2}\left(\alpha_{2}\right) l_{2}+\mathcal{G}\left(\alpha_{1}\right) l_{1} \gamma \\
m v(\dot{\psi}+\dot{\beta}) & =\mathbf{F}_{\text {lat, } \mathbf{1}}\left(\alpha_{1}\right)+\mathbf{F}_{\text {lat }, \mathbf{2}}\left(\alpha_{2}\right)= \\
& =\mathcal{F}_{1}\left(\alpha_{1}\right)+\mathcal{F}_{2}\left(\alpha_{2}\right)+\mathcal{G}\left(\alpha_{1}\right) \gamma
\end{aligned}
$$

where $m$ is the mass of the vehicle, $J$ is yaw-inertia, $l_{1}$ and $l_{2}$ are geometric parameters. $\beta$ is the side-slip angle of the chassis, $\dot{\psi}$ is yaw-rate. $\mathbf{F}_{\text {lat, } \mathbf{1}}\left(\alpha_{1}\right)$ and $\mathbf{F}_{\text {lat, } \mathbf{2}}\left(\alpha_{2}\right)$ represent lateral tire forces, which depend on tire side-slip angles $\alpha_{1}$ and $\alpha_{2}$.

In the case of the variable-geometry suspension system two nonlinearities of the tire characteristics must be considered in a given operation range.

- Lateral tire force $\mathcal{F}(\alpha)$ depends on the lateral tire slip $\alpha$ nonlinearly. Although in several control applications the lateral forces are approximated with linear functions, which results a simple description, it can be used in a narrow tire side-slip range. Vehicle motion is significantly characterized by this nonlinearity.

- The generated lateral tire force from camber angle $\mathcal{G}(\alpha)$ depends on $\alpha$ nonlinearly. Thus, the efficiency of actuator intervention is influenced by tire slip.

The nonlinear model of the tire is constructed from the polynomial approximation of the previous two effects, $\mathcal{F}(\alpha)$ and $\mathcal{G}(\alpha)$ :

$$
\mathbf{F}_{\text {lat }}(\alpha)=\mathcal{F}(\alpha)+\mathcal{G}(\alpha) \gamma=\sum_{j=1}^{n} c_{j} \alpha^{j}+\sum_{k=0}^{m} g_{k} \alpha^{k} \gamma
$$

where $\gamma$ is the camber angle of the wheel. An example of

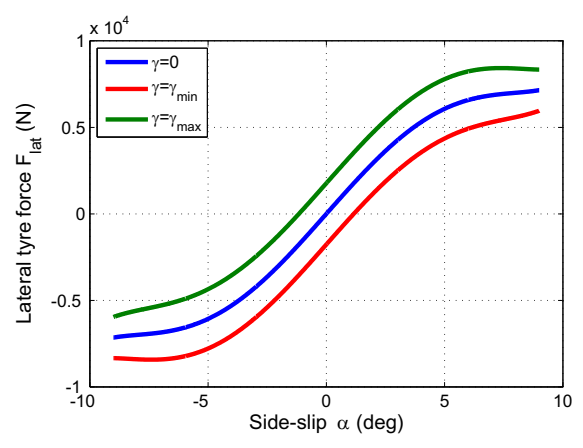

Fig. 3. Modeling of lateral tire force $\mathbf{F}_{\text {lat }}$

the nonlinear characteristics in the function of tire side-slip $\alpha$ is illustrated in Figure 3. 
The relationships between the tire side-slip angles for the front and rear axles, the steering angle of the vehicle and the side-slip angle of the chassis are $\tan \left(\delta-\alpha_{1}\right)=\left(l_{f} \dot{\psi}+\right.$ $v \sin \beta) /(v \cos \beta)$ and $\tan \left(\alpha_{2}\right)=\left(l_{r} \dot{\psi}-v \sin \beta\right) /(v \cos \beta)$. At stable driving conditions the tire side-slip angle $\alpha_{i}$ is normally not greater than $10^{\circ}$ and the equations can be simplified by substituting $\sin \beta \approx \beta$ and $\cos \beta \approx 1$. Moreover, the relative error of these simplifications is less than $1 \%$. Thus, the following side-slip angles of the front and rear axles can be approximated: $\alpha_{1}=\delta-\beta-\dot{\psi} l_{1} / v$ and $\alpha_{2}=-\beta+\dot{\psi} l_{2} / v$. In the followings these expressions are used to transform (1) into a polynomial state-space representation $\dot{x}=f(x)+g u$, where $x$ is the state vector, $u$ is the control input signal, $f$ and $g$ are matrices.

From the above equations the yaw-rate and side-slip of the vehicle can be expressed in the following forms:

$$
\dot{\psi}=v \frac{\alpha_{2}-\alpha_{1}+\delta}{l_{1}+l_{2}}, \quad \beta=-\frac{\alpha_{1} l_{2}+\alpha_{2} l_{1}-l_{2} \delta}{l_{1}+l_{2}}
$$

Since (1) contains the time-derivatives of $\dot{\psi}$ and $\beta$, they must be differentiated by using (3). At constant velocity $v$ their derivatives are:

$$
\ddot{\psi}=v \frac{\dot{\alpha}_{2}-\dot{\alpha}_{1}+\dot{\delta}}{l_{1}+l_{2}}, \quad \dot{\beta}=-\frac{\dot{\alpha}_{1} l_{2}+\dot{\alpha}_{2} l_{1}-l_{2} \dot{\delta}}{l_{1}+l_{2}}
$$

Now the vehicle model (1) is reformulated:

$$
\begin{aligned}
\dot{\alpha}_{2}-\dot{\alpha}_{1}= & {\left[\frac{l_{1}+l_{2}}{J v}\left(\mathcal{F}_{1}\left(\alpha_{1}\right) l_{1}-\mathcal{F}_{2}\left(\alpha_{2}\right) l_{2}\right)\right]-} \\
& -\dot{\delta}+\frac{l_{1}\left(l_{1}+l_{2}\right)}{J v} \mathcal{G}\left(\alpha_{1}\right) \gamma \\
\dot{\alpha}_{1} l_{2}+\dot{\alpha}_{2} l_{1}= & v\left(\alpha_{2}-\alpha_{1}\right)-\frac{l_{1}+l_{2}}{m v}\left[\mathcal{F}_{1}\left(\alpha_{1}\right)+\mathcal{F}_{2}\left(\alpha_{2}\right)\right]+ \\
& +v \delta+l_{2} \dot{\delta}-\frac{l_{1}+l_{2}}{m v} \mathcal{G}\left(\alpha_{1}\right) \gamma
\end{aligned}
$$

The rearrangement of vehicle model shows, that the new states of the model are tire slip angles $\alpha_{1}$ and $\alpha_{2}$. In this way the nonlinearity of the lateral tire forces $\mathcal{F}_{1}, \mathcal{F}_{2}$ and $\mathcal{G}$ can be considered. However, (5) incorporates the timederivative of the front-wheel steering angle. In the actuator efficiency analysis the limit of intervention $\max (|\delta|)$ has relevance. The detailed vehicle model is used for actuation range determination. For actuation limit purposes the following approximations are applied:

$$
\begin{aligned}
\max (|\dot{\delta}|) & =\max \left(\frac{|\dot{\delta}|}{|\delta|}\right) \cdot \max (|\delta|)=\nu \cdot \max (|\delta|), \\
\dot{\delta} & \approx \nu \cdot \delta,
\end{aligned}
$$

where parameter $\nu$ represents the relationship between the maximum steering value and the variation speed of $\delta$. Since $\max \delta$ is a given fixed limit at the actuator analysis, high $\nu$ value represents a fast changing steering signal, while a slow changing steering signal is modeled with low $\nu$. Note that the proposed modeling formula is only valid for actuation limit computation.

The polynomial state-space representation of the system is formulated using (5) and the substitution of (6) is as below:

$$
\dot{x}=\left[\begin{array}{c}
\dot{\alpha}_{1} \\
\dot{\alpha}_{2}
\end{array}\right]=\left[\begin{array}{c}
\mathrm{f}_{1}\left(\alpha_{1}, \alpha_{2}\right) \\
\mathrm{f}_{2}\left(\alpha_{1}, \alpha_{2}\right)
\end{array}\right]+\left[\begin{array}{l}
h_{1} \\
h_{2}
\end{array}\right] \delta+\left[\begin{array}{l}
g_{1} \\
g_{2}
\end{array}\right] \gamma
$$

where

$$
\begin{aligned}
\mathrm{f}_{1}= & \frac{l_{1}}{J v}\left[\mathcal{F}_{2}\left(\alpha_{2}\right) l_{2}-\mathcal{F}_{1}\left(\alpha_{1}\right) l_{1}\right]+ \\
& +\frac{v}{l_{1}+l_{2}}\left(\alpha_{2}-\alpha_{1}\right)-\frac{1}{m v}\left[\mathcal{F}_{1}\left(\alpha_{1}\right)+\mathcal{F}_{2}\left(\alpha_{2}\right)\right] \\
\mathrm{f}_{2}= & \frac{l_{2}}{J v}\left[\mathcal{F}_{1}\left(\alpha_{1}\right) l_{1}-\mathcal{F}_{2}\left(\alpha_{2}\right) l_{2}\right]+ \\
& +\frac{v}{l_{1}+l_{2}}\left(\alpha_{2}-\alpha_{1}\right)-\frac{1}{m v}\left[\mathcal{F}_{1}\left(\alpha_{1}\right)+\mathcal{F}_{2}\left(\alpha_{2}\right)\right] \\
h_{1}= & \frac{v}{l_{1}+l_{2}}+\nu, \quad h_{2}=\frac{v}{l_{1}+l_{2}} \\
g_{1}= & -\left(\frac{l_{1}^{2}}{J v}+\frac{1}{m v}\right) \mathcal{G}\left(\alpha_{1}\right), \quad g_{2}=\left(\frac{l_{1} l_{2}}{J v}-\frac{1}{m v}\right) \mathcal{G}\left(\alpha_{1}\right)
\end{aligned}
$$

\subsection{Modeling of variable-geometry suspension construction}

In the following the relationship between $\delta_{c}$ and $\gamma$ is proposed. The scheme of the variable-geometry suspension system is illustrated in Figure 4. The $a_{y}$ modification of the lateral position of $A$ affects the rotation of the front wheel around axis $B K$. Thus, the camber angle $\gamma$ and steering angle $\delta_{c}$ are simultaneously changed. The position of the steering track-rod connection point $K$ has an important role in the distribution of $\delta_{c}$ and $\gamma$. Thus, it is necessary to choose a vertical position $K_{z}$, by which the lateral force on the tire is improved the most effectively.

The deduction of the relationships $\delta_{c}=\mathfrak{f}_{\delta_{c}}\left(a_{y}, K_{z}\right)$ and $\gamma=\mathfrak{f}_{\gamma}\left(a_{y}, K_{z}\right)$ is detailed in Németh and Gáspár [2013b]. In the following the applied relations are proposed, which have been constructed using the SimMechanics toolbox of Matlab. In the model the arms and bodies of the system are elements which are connected to the vehicle chassis by joints. Figures 5(a), 5(b) and 5(c) show angles $\delta_{c}, \gamma$ and half-track change $\Delta B$ at different $K_{z}$ heights. The aim of the example is to illustrate the relationship between signals. The variation of $K_{z}$ has a great influence on angle $\delta_{c}$ and it modifies $\gamma$ slightly. $K B$ is the axis of wheel rotation during the actuation $a_{y}$, therefore its orientation influences the relationship between these angles. Since generally $\delta_{c}$ and $\gamma$ are in conflict, it is necessary to find an appropriate solution to parameter $K_{z}$. In the analyzed construction $K_{z}$ has a significant influence on $\delta_{c}$ and with an increased $K_{z}$ it is possible to achieve high lateral tire force. Moreover, $K_{z}$ influences the lateral movement of

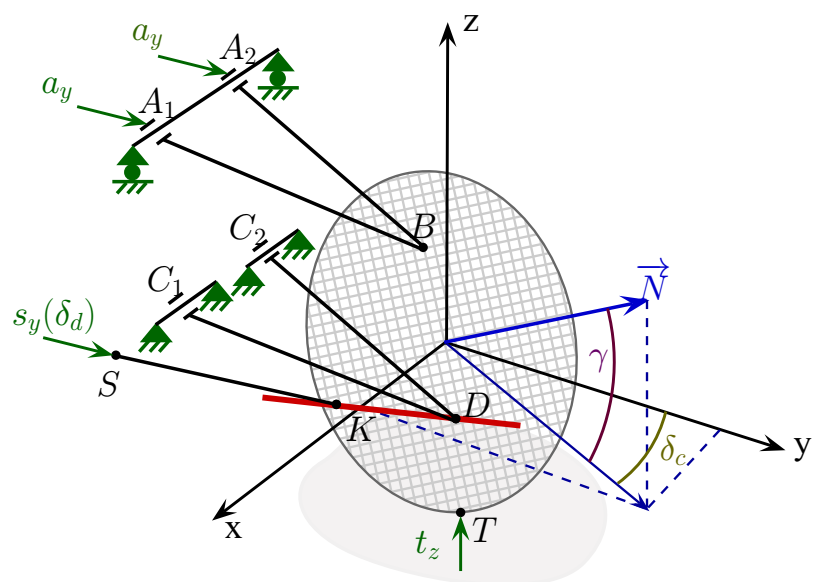

Fig. 4. Wheel position related to the steering and the camber angle 
$T$, i.e., the half-track change which is denoted by $\Delta B$. It has an important role in tire wear. Consequently, the steering angle, the camber angle and the half-track change are functions of the actuation, i.e., $\delta_{c}=\mathfrak{f}_{\delta_{c}}\left(a_{y}\right), \gamma=\mathfrak{f}_{\gamma}\left(a_{y}\right)$ and $\Delta B=\mathfrak{f}_{\Delta B}\left(a_{y}\right)$. The presented example also shows the conflict between the three parameters. A high $K_{z}$ prefers $\delta_{c}$, while it is disadvantageous for $\gamma$.

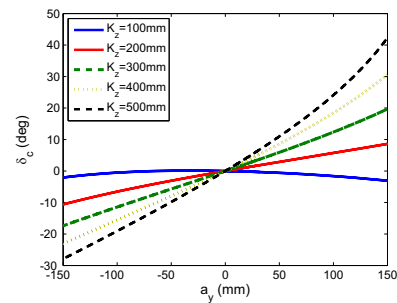

(a) $\delta_{c}$ change

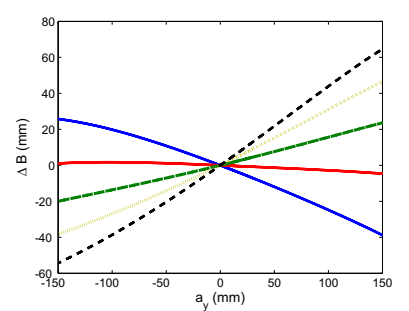

(c) $\Delta B$ change

Fig. 5. Influence of $K_{z}$ on the relationship between $\delta_{c}, \gamma$ and $\Delta B$

\section{NONLINEAR APPROACH FOR ACTUATOR EFFICIENCY ANALYSIS}

In this section the fundamental concepts of the Sumof-Squares (SOS) programming are introduced, which is a suitable method to analyze and control a nonlinear polynomial systems. The method is applied to determine actuator limit influences in vehicle dynamics.

\subsection{Theoretical background}

Several papers deal with SOS programming, which has been elaborated in the past decade for control purposes. Important theorems in SOS programming was proposed in Parrilo [2003]. Prajna et al. [2004] showed sufficient conditions for the solutions to nonlinear control problems, which are formulated in terms of state dependent Linear Matrix Inequalities (LMI). Jarvis-Wloszek et al. [2003] introduced the application of SOS programming to several control problems, e.g. reachable set computation and control design algorithm. The local stability analysis of polynomial systems and an iterative computation method for their region of attraction were presented in Tan and Packard [2008]. In Scherer and Hol [2006] the SOS method was applied to non-convex problems. Robust performance in polynomial control systems was analyzed in Topcu and Packard [2009]. As a new result the maximum controlled invariants sets of polynomial control systems were calculated in Korda et al. [2013].

The following definitions are essential to understand SOS programming Jarvis-Wloszek et al. [2003]. The basic el- ements of the method are polynomials and SOS as defined below: A Polynomial $f$ in $n$ variables is a finite linear combination of the functions $m_{\alpha}(x):=x^{\alpha}=$ $x_{1}^{\alpha_{1}} x_{2}^{\alpha_{2}} \cdots x_{n}^{\alpha_{n}}$ for $\alpha \in \mathbb{Z}_{+}^{n}$, deg $m_{\alpha}=\sum_{i=1}^{n} \alpha_{i}$ :

$$
f:=\sum_{\alpha} c_{\alpha} m_{\alpha}=\sum_{\alpha} c_{\alpha} x^{\alpha}
$$

with $c_{\alpha} \in \mathbb{R}$. Define $\mathbb{R}$ to be the set of all polynomials in $n$ variables. The degree of $f$ is defined as $f:=\max _{\alpha} \operatorname{deg} m_{\alpha}$. The set of Sum-of-Squares (SOS) polynomials in $n$ variables is defined as:

$$
\Sigma_{n}:=\left\{p \in \mathcal{R}_{n} \mid p=\sum_{i=1}^{t} f_{i}^{2}, f_{i} \in \mathcal{R}_{n}, i=1, \ldots, t\right\}
$$

The goal of the nonlinear actuator analysis is the determination of their intervention limits next to a peakbounded actuation. With an appropriate intervention of the actuators some of the instable regions can be stabilized. In the next section an answer to the following question is sought: how much is the largest state-space region where the stability of the system can be guaranteed by a given peak-bounded control input? This question leads to the computation of Controlled Invariant Sets.

\subsection{Computation method of Controlled Invariant Sets}

The state-space representation of the system is given in the next form, see (8):

$$
\dot{x}=f(x)+g u
$$

where $f(x)$ is a matrix, which incorporates smooth polynomial functions and $f(0)=0$. In the next analysis one control input is considered, thus $u=M_{b r}$ or $u=\delta$. The global asymptotical stability of the system at the origin is guaranteed by the existence of the Control Lyapunov Function of the system defined as follows Sontag [1989]: $A$ smooth, proper and positive-definite function $V: \mathbb{R}^{n} \rightarrow \mathbb{R}$ is a Control Lyapunov Function for system if

$$
\inf _{u \in \mathbb{R}}\left\{\frac{\partial V}{\partial x} f(x)+\frac{\partial V}{\partial x} g \cdot u\right\}<0
$$

for each $x \neq 0$. According to Definition 3.2 two cases are differentiated:

- if $\frac{\partial V}{\partial x} f(x)<0$ then the system is stable and $u \equiv 0$. This stability scenario is contained by the next two stability criteria.

- if $\frac{\partial V}{\partial x} f(x)>0$ then the system is unstable. However, the system can be stabilized

- if $\frac{\partial V}{\partial x} g<0$ and $\frac{\partial V}{\partial x} f(x)+\frac{\partial V}{\partial x} g \cdot u_{\max }<0$, the upper peak-bound of control input $u$ stabilizes the system.

- if $\frac{\partial V}{\partial x} g>0$ and $\frac{\partial V}{\partial x} f(x)-\frac{\partial V}{\partial x} g \cdot u_{\max }<0$, the lower peak-bound of control input $u$ stabilizes the system. Note that $u_{\min }=-u_{\max }$.

The Controlled Invariant Set of the system (11) is defined as the level-set of the Control Lyapunov Function at $V(x)=1$. Thus, the fulfilment of the previous stability criterion must be guaranteed at $V(x) \leq 1$.

In the following an iterative computation method is proposed to find the maximum Controlled Invariant Set, which can lead to an easier calculation according to our experience. The practical method contains three steps: 
Step 1: The region of attraction of the uncontrolled system $\dot{x}=f(x)$ is determined as an initial set. In this step the maximum level set of $V_{0}=1$ is found, which is incorporated in the stabile region. The SOS based computation of region of attraction was presented in Jarvis-Wloszek [2003].

Step 2: An $\eta$ parameter is chosen and $V_{\eta}=V_{0} \cdot \eta$ is checked as a Local Control Lyapunov Function. The levelset $V_{\eta}=1$ represents a Controlled Invariant Set $S_{\eta}$, in which the system can be stabilized using a finite control input $u$. Depending on parameter $\eta$ the size of the level-set can be enlarged or reduced. The SOS based computation of Local Control Lyapunov Function is proposed in Tan and Packard [2008].

Step 3: In the final step the acceptability and the enlarging possibility of $S_{\eta}$ Controlled Invariant Set must be checked. The peak-bounds of the actuation are $u_{\min }=$ $-u_{\max }$ and $u_{\max } . S_{\text {inst }}=\frac{\partial V}{\partial x} f(x)>0$ is the instable region of the system. $S_{\min }=\frac{\partial V}{\partial x} f(x)-\frac{\partial V}{\partial x} g \cdot u_{\max }>0$ is the region, which can not be stabilized by $u_{\text {min }}$. Similarly, $S_{\max }=\frac{\partial V}{\partial x} f(x)+\frac{\partial V}{\partial x} g \cdot u_{\max }>0$ is the region, which can not be stabilized by $u_{\max }$. If $S_{\eta}$ is an appropriate Controlled Invariant Set and $V_{\eta}$ is an appropriate Control Lyapunov Function, then

$$
S_{\eta} \bigcap S_{i n s t} \bigcap S_{\min } \bigcap S_{\max }=\emptyset
$$

The emptiness of the intersection condition defined below can be checked manually by the plot of $S_{\eta}, S_{\text {inst }}, S_{\text {min }}$ and $S_{\text {max }}$. Additionally, if $S_{\eta}$ is appropriate then $\eta$ value can be reduced in the previous step to maximize the Controlled Invariant Set.

\section{DEMONSTRATION OF ACTUATOR EFFICIENCY}

In the following an example of Maximum Controlled Invariant Set $\mathbf{S}$ analysis is proposed. Since wheel tilting has a great impact in the design of lightweight vehicles, in the example a small vehicle with double-wishbone suspension is considered, see Table 1.

Table 1. Data of vehicle model

\begin{tabular}{|c|c||c|c||c|c|}
\hline$m$ & $800 \mathrm{~kg}$ & $c_{1,1}$ & 827.6 & $c_{1,2}$ & 551.8 \\
\hline$J$ & $300 \mathrm{~km}^{2}$ & $c_{2,1}$ & -3.315 & $c_{2,2}$ & -2.21 \\
\hline$l_{2}$ & $1.1 \mathrm{~m}$ & $c_{3,1}$ & -8.211 & $c_{3,2}$ & -5.474 \\
\hline$l_{1}$ & $0.9 m$ & $c_{4,1}$ & 0.133 & $c_{4,2}$ & 0.089 \\
\hline$\nu$ & 20 & $c_{5,1}$ & 0.04 & $c_{5,2}$ & 0.027 \\
\hline$g_{0}$ & 100 & $c_{6,1}$ & -0.0012 & $c_{6,2}$ & $-8.2 \cdot 10^{-4}$ \\
\hline$g_{4}$ & -0.005 & & & & \\
\hline
\end{tabular}

The form of the polynomial tire model is the following, see (2):

$$
\begin{aligned}
\mathcal{F}_{1}\left(\alpha_{1}\right) & =\sum_{j=1}^{6} c_{j, 1} \alpha_{1}^{j}, \quad \mathcal{F}_{2}\left(\alpha_{2}\right)=\sum_{j=1}^{6} c_{j, 2} \alpha_{2}^{j}, \\
\mathcal{G}\left(\alpha_{1}\right) & =g_{0}+g_{4} \alpha_{1}^{4} .
\end{aligned}
$$

In the analysis the influence of $K_{z}$ on the Maximal Controlled Invariant Sets is examined. The maximal control input of the variable-geometry suspension actuator is $\left|a_{y, \max }\right|=150 \mathrm{~mm}$, see Figure 5. Two scenarios are compared in the example: in the first case the variablegeometry suspension with different $K_{z}$ parameters is considered, the dynamics of the vehicle is influenced during steering and tilting effects. In the second case the front wheel steering system with steering angle limitation $\left|\delta_{\text {max }}\right|$ is supposed, where $\left|\delta_{\max }\right|$ is equal to the maximal steering effect of the variable-geometry suspension, see Figure 5(a).

The Maximum Controlled Invariant Sets at different velocities and $K_{z}=300 \mathrm{~mm},\left|\delta_{\max }\right|=18^{\circ}$ are illustrated in Figure 6. Since the instable regions of the lateral dy-

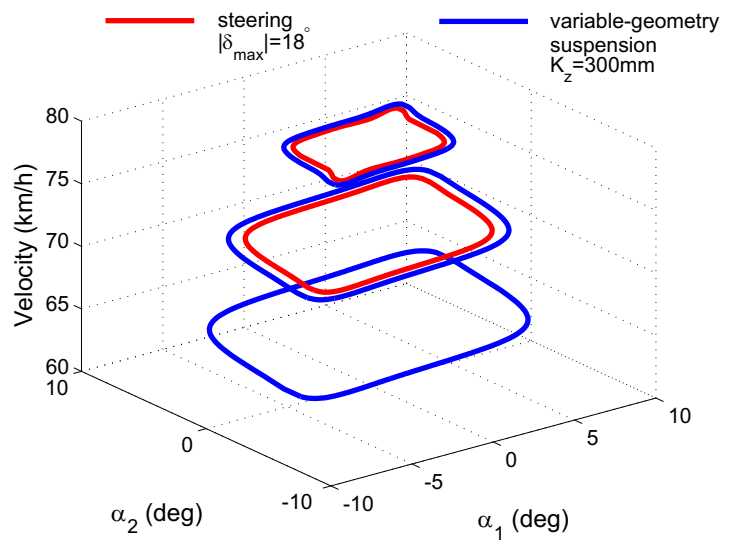

Fig. 6. Maximal Controlled Invariant Sets of the systems

namics increase at higher velocity, the controllable region with limited control input is reduced. At $v=65 \mathrm{~km} / \mathrm{h}$ the steering and the suspension sets reach the limit of model validity, thus the wheel camber angle has relevance at $v>65 \mathrm{~km} / \mathrm{h}$. During the simultaneous actuation of steering and tilting, $\mathbf{S}$ regions are enlarged compared to the individual steering. In Figure 6 the distance of set boundary from zero increases approximately 10\%. When the results are compared with Figure 1 (which is calculated using the same parameters) the efficiency of the proposed set computation method is conspicuous: the increase of instable regions and the benefit of variable-geometry suspension are demonstrated.

In Figure 7 the Maximal Controlled Invariant Sets of each $K_{z}$ value are compared at fixed velocities. When $K_{z}=100 \mathrm{~mm}$ the wheel tilting intervention is dominant, while steering has a slight counter-influence. Thus, $K_{z}=$ $100 \mathrm{~mm}$ can be analyzed in variable-geometry suspension, because pure steering is inefficient. $K_{z}=300 \mathrm{~mm}$ leads to a balance between the camber and the steering angle, while at $K_{z}=500 \mathrm{~mm}$ steering is preferred.

Figure 7 also shows that construction parameter $K_{z}=$ $100 \mathrm{~mm}$ results in a small $\mathbf{S}$ region at both velocities. Thus, camber angle intervention is insufficient by itself, so it is necessary to find a $K_{z}$, which causes $\gamma$ and $\delta_{c}$ angles simultaneously. It can be seen that the simultaneous actuation of steering and wheel tilting leads to the enlargement of $\mathbf{S}$, see $K_{z}=300 \mathrm{~mm}$ and $K_{z}=500 \mathrm{~mm}$ scenarios. However, the enlargement is more significant at $K_{z}=300 \mathrm{~mm}$. Moreover, $\mathbf{S}$ region of $K_{z}=300 \mathrm{~mm}$ is larger than the region of $K_{z}=500 \mathrm{~mm}$. The reason for these phenomena is the reduction of the maximal $\gamma$ at high $K_{z}$ values.

The results of the analysis show that $K_{z}$ influences the Maximum Controlled Invariant Sets significantly. The minimization of $\gamma$ or $\delta_{c}$ leads to small sets, thus it is 


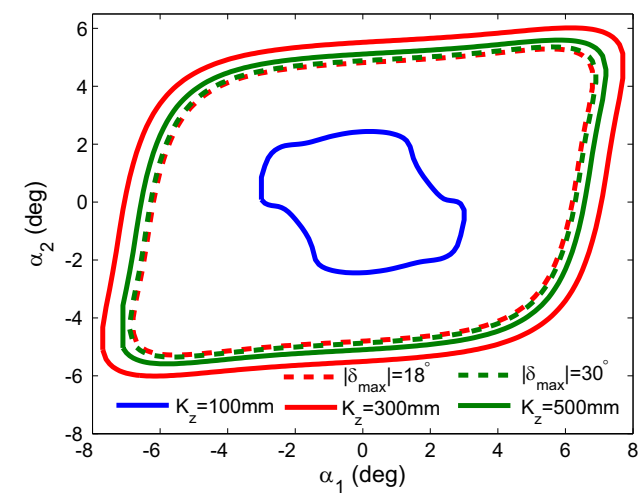

(a) $v=72 \mathrm{~km} / \mathrm{h}$

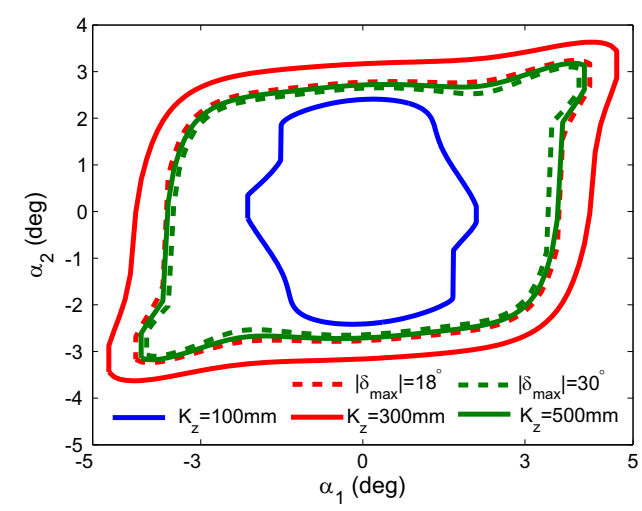

(b) $v=80 \mathrm{~km} / \mathrm{h}$

Fig. 7. Maximal Controlled Invariant Sets

required to find a balance between the tilting and steering effects of variable-geometry suspension. Another important performance of the system is half-track change $\Delta B$. Thus, the following criterion results in an optimal $K_{z}$ height of suspension construction:

$$
\min _{K_{z} \in \mathcal{K}_{z}}\left(\sum_{i=v_{\min }}^{v_{\max }} \frac{1}{A r\left(\mathbf{S}_{i}\left(K_{z}\right)\right)}+Q \int_{-a_{y, \max }}^{a_{y, \max }}\left|\Delta B\left(K_{z}, a_{y}\right)\right| \mathrm{d} a_{y}\right)
$$

where $\mathcal{K}_{z}$ is the set of possible $K_{z}$ values, $v_{\max }$ and $v_{\text {min }}$ are the minimal and maximal velocity values in the region of investigation, $a_{y, \max }$ is the intervention limit of the variable-geometry suspension. $\operatorname{Ar}\left(\mathbf{S}_{i}\left(K_{z}\right)\right)$ is the area of the $i^{t h}$ Maximal Controlled Invariant Set. $Q$ is a design parameter, which guarantees a balance between the maximization of $A r\left(\mathbf{S}_{i}\left(K_{z}\right)\right)$ and the minimization of $\Delta B$.

\section{CONCLUSION}

In the paper the steering and wheel tilting abilities of the variable-geometry suspension have been analyzed. The effects of the camber angle $\gamma$ and steering angle $\delta_{c}$ on the operation regions have been analyzed based on the nonlinear polynomial SOS programming method. The analysis has provided important information for the controlled invariant sets and, thus the functional reconfiguration possibilities of the actuators.

\section{REFERENCES}

C. Canudas de Wit, H. Olsson, K. J. Astrom, and P. Lischinsky. A new model for control of systems with friction. IEEE Trans. Automatic Control, 40(3):419-425, 1995.

H. Dugoff, P. S. Fancher, and L. Segel. Tire performance characteristics affecting vehicle response to steering and braking control inputs. Final report, 1969.

W.J. Evers, A. van der Knaap, I. Besselink, and H. Nijmeijer. Analysis of a variable geometry active suspension. Int. Symp. Advanced Vehicle Control, Kobe, Japan, 2008.

M. S Fallah, R. Bhat, and W. F. Xie. New model and simulation of macpherson suspension system for ride control applications. Vehicle System Dynamics, 47(2):195-220, 2009.

A. Goodarzia, E. Oloomia, and E. Esmailzadehb. Design and analysis of an intelligent controller for active geometry suspension systems. Vehicle System Dynamics, 49(1):333-359, 2010.

Z. Jarvis-Wloszek. Lyapunov Based Analysis and Controller Synthesis for Polynomial Systems using SOS Optimization. Ph.D. Thesis, University of California, Berkeley, 2003.

Z. Jarvis-Wloszek, R. Feeley, W. Tan, K. Sun, and A. Packard. Some controls applications of sum of squares programming. 42nd IEEE Conference on Decision and Control, Maui, 5:4676-4681, 2003.

U. Kiencke and L. Nielsen. Automotive control systems for engine, driveline and vehicle. Springer, 2000.

M. Korda, D. Henrion, and C. N. Jones. Convex computation of the maximum controlled invariant set for polynomial control systems. 2013.

U. K. Lee, S. H. Lee, C. S. Han, K. Hedrick, and A. Catala. Active geometry control suspension system for the enhancement of vehicle stability. Proc. IMechE, Part D: J. Automobile Engineering, 222(6):979-988, 2008.

B. Németh and P. Gáspár. Mechanical analysis and control design of mcpherson suspension. Int. J. Vehicle Systems Modelling and Testing, 7:173 - 193, 2012.

B. Németh and P. Gáspár. Control design of variable-geometry suspension considering the construction system. IEEE Transactions on Vehicular Technology, 2013a.

B. Németh and P. Gáspár. Variable-geometry suspension design in driver assistance systems. 12nd European Control Conference, Zürich, pages 1481-1486, $2013 \mathrm{~b}$.

H. B. Pacejka. Tyre and vehicle dynamics. Elsevier ButterworthHeinemann, Oxford, 2004.

P. Parrilo. Semidefinite programming relaxations for semialgebraic problems. Mathematical Programming B, 96(2):293-320, 2003.

D. Piyabongkarn, T. Keviczky, and R. Rajamani. Active direct tilt control for stability enhancement of a narrow commuter vehicle. Int. J. Automotive Technology, 5(2):77-88, 2004.

S. Prajna, A. Papachristodoulou, and F. Wu. Nonlinear control synthesis by sum of squares optimization: A Lyapunov-based approach. 5th IEEE Asian Control Conference, 1:157-165, 2004.

R. Sancibrian, P. Garcia, F. Viadero, and A. Fernandez. Kinematic design of double-wishbone suspension systems using a multiobjective optimisation approach. Vehicle System Dynamics, 48(7): 793-813, 2010.

C. W. Scherer and C. W. J. Hol. Matrix sum-of-squares relaxations for robust semi-definite programs. Math. Program, 107:189-211, 2006 .

E.D. Sontag. A "universal" construction of Artstein's theorem on nonlinear stabilization. Systems \& Control Letters, 13:117-123, 1989.

L. Suarez. Active Tilt and Steer Control for a Narrow Tilting Vehicle: Control design and implementation. Lambert Academic Publishing, 2012.

W. Tan and A. Packard. Stability region analysis using polynomial and composite polynomial Lyapunov functions and sum-ofsquares programming. IEEE Trans. Automatic Control, 53(2): 565-571, 2008.

U. Topcu and A. Packard. Local robust performance analysis for nonlinear dynamical systems. Proc. American Control Conference, pages 784-789, 2009. 